\title{
Improving Immersive Experiences for Visitors with Sensory Impairments to the Aquarium of the Pacific
}

\begin{tabular}{|c|c|}
\hline Bríd O’Conaill & Daniel Hajas \\
\hline Ultraleap & SCHI Lab, University of Sussex \\
\hline $\begin{array}{l}\text { Bristol, BS2 0EL, UK } \\
\text { brid.oconaill@ultraleap.com }\end{array}$ & $\begin{array}{l}\text { Brighton, BN1 9RH, UK } \\
\text { dh256@sussex.ac.uk }\end{array}$ \\
\hline James Provan & Marianna Obrist \\
\hline Ultraleap & SCHI Lab, University of Sussex \\
\hline $\begin{array}{l}\text { Bristol, BS2 0EL, UK } \\
\text { james.provan@ultraleap.com }\end{array}$ & $\begin{array}{l}\text { Brighton, BN1 9RH, UK } \\
\text { m.obrist@sussex.ac.uk }\end{array}$ \\
\hline Jerry Schubel & Loïc Corenthy \\
\hline Aquarium of the Pacific & Ultraleap \\
\hline $\begin{array}{l}\text { Long Beach, CA 90802, USA } \\
\text { jschubel@lbaop.org }\end{array}$ & $\begin{array}{l}\text { Bristol, BS2 0EL, UK } \\
\text { loic.corenthy@ultraleap.com }\end{array}$ \\
\hline
\end{tabular}

Permission to make digital or hard copies of part or all of this work for personal or classroom use is granted without fee provided that copies are not made or distributed for profit or commercial advantage and that copies bear this notice and the full citation on the first page. Copyrights for third-party components of this work must be honored. For all other uses, contact the owner/author(s).

CHI '20 Extended Abstracts, April 25-30, 2020, Honolulu, HI, USA.

Copyright is held by the author/owner(s).

ACM ISBN 978-1-4503-6819-3/20/04.

http://dx.doi.org/10.1145/3334480.3375214

\begin{abstract}
This case study describes the development of a mid-air haptic solution to enhance the immersive experience of visitors who are deaf, blind or wheelchair users to the Aquarium of the Pacific's movie theatre. During the project we found that adding a sense of touch, using an innovative ultrasound technology, to an immersive experience can improve the sense of engagement users have with the content, and can help to improve agreement with the topics presented. We present guidelines on the design of haptic sensations. By describing how this project took place within the tight timelines of a commercial deployment, we hope to encourage more organisations to do similar work.
\end{abstract}

\section{Author Keywords}

Immersive experiences, Multimodal, Haptic feedback, Sensory impaired

\section{CCS Concepts}

-Human-centered computing $\rightarrow$ User studies; Haptic devices; Accessibility design and evaluation methods;

\section{Introduction}

The Aquarium of the Pacific (the Aquarium) is the fourth most attended aquarium in the USA with a mission to "instill a sense of wonder, respect, and stewardship for the Pacific Ocean, its inhabitants, and ecosystems". Its vision is to 


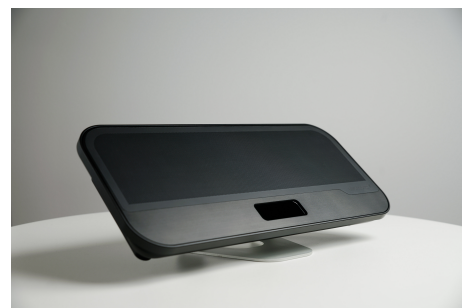

Figure 1: STRATOS Inspire by Ultraleap

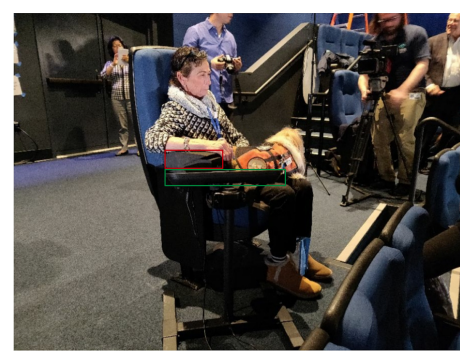

Figure 2: A participant during the Exploration user study (WP1). The STRATOS Inspire is highlighed in green and the props in red. "create an aquarium dedicated to conserving and building Natural Capital (Nature and Nature's services) by building Social Capital (the interactions between and among peoples)" [1].

The Aquarium built Pacific Visions, a new building, enabling the public to experience "a state-of-the-art immersive theater, interactive art installations, engaging multimedia displays, and live animal exhibits." Committed to going beyond the requirements of the Americans with Disabilities Act (ADA) and seeking a way to augment the movie experience for visitors who may have a sensory impairment, The Aquarium commissioned Ultraleap (UL) to provide a tactile experience to complement the movie. Wheelchair users were also included in the brief, as they would not be able to feel the effects of the rumble seats.

The project took place over a four month period with strict deadlines corresponding to Pacific Visions opening dates. To the best of our knowledge, this is a world first; augmenting a multisensory experience with mid-air haptics, for users with a sensory impairment, in a public deployment.

\section{Background}

Immersive experiences

Sun Joo Ahn et al. [4] showed the effectiveness of immersive VR experiences in communicating environmental themes and subsequently influenced real-life behaviour. For environmental organisations like The Aquarium, this suggests immersive experiences are a useful way to engage their visitors and promote their vision. Immersive movie experiences are an attractive alternative to VR for organisations that have to accommodate a large number of visitors. New technologies such as Ultraleap's mid-air haptics make it possible to consider tactile stimulation as an additional ex- perience for users who may not be able to access all the sensory effects.

The design of complementary haptic experiences is, however, not a straightforward task. The principle of equipotentiality; "the idea that the same type of touch can be assigned very different meanings or consequences", mentioned by Hertenstein et al. [6], is a concern. It raises the possibility that any design of the haptic modality, no matter how it correlates to the audio-visual content, won't necessarily be perceived in a similar manner by different users.

Mid-air haptics and audio-visual experiences

UL technology and the creation of mid-air haptics is described in [5]. Ultrasound is emitted from an array of ultrasonic transducers and focused on to a person's hand, (palm and/or fingers), to create tactile sensations. The hardware used in this project, a STRATOS Inspire, is shown in Figure 1 (the transducers are located behind the cover material and not visible in this picture).

Previous studies have investigated the use of haptic feedback to enhance audiovisual experiences, both within an instrumented-hand setup or a free mid-air interaction setup. Ablart et al. [2] evaluated the sensory augmentation of audiovisual content using mid-air haptic feedback. They targeted short, i.e. one-minute long, movie experiences and reported on the effect of generic haptic patterns with respect to their temporal integration with the movie. No significant effect of the synchronisation of the haptic feedback with the content of the movie was found. In contrast, our studies found synchronising the haptic feedback with the audiovisual content (including audio description) seems to be a key aspect of creating compelling experiences. We explore possible reasons for this in the Discussion section. 
Another interesting aspect is the influence of prior knowledge on users while experiencing a new type of multi-modal interaction. Mayer's cognitive theory of multimedia learning [8] takes prior knowledge into account to explain how users learn from multimedia stimuli. Jones et al. [7] investigated how the introduction of haptic feedback can influence this model.

Haptics and accessibility

Haptic interfaces have been used in projects aiming to improve different interaction aspects for blind users. Nam et al. [9] developed a learning system, the Molecular Property Module, proposing different types of learning interfaces controlled via a Novint Falcon haptic device ${ }^{0}$. They suggest useful design guidelines and principles such as the importance of training for first time users or the dangers of over-stimulation and sensory overload that were applicable in this study.

Haptic interfaces have also been used in attempts to improve the experience of hearing impaired users when interacting with multimedia content, notably music. When experiencing music, people can feel sound vibrations through different parts of their body. This is especially important for users with a hearing impairment. The Haptic Chair, Nanayakkara et al. [10], amplifies the natural vibrations produced by music and conveys them to users through the haptic channel. Our setup is similar to the Haptic Chair in the sense that users will have a passive experience, seated in a chair, and be able to perceive complementary information through the haptic channel, mainly via their resting hands.

\section{Method}

We approached the project by breaking it down into three Work Packages (WP), each centred around a user study: i)
Exploration, ii) Implementation, iii) Final delivery. This case study focuses on the first two as the final work package was primarily concerned with ensuring the physical integration worked and that the experience maintained value as part of a larger multisensory show.

\section{WP1: Exploration}

Six participants, four male and two female, (ages unrecorded), were recruited by the Aquarium as representative of their target audience. Four participants were deaf, two participants were blind, (one of whom was autistic and a musical savant). None of them had previous experience with UL technology. We began by allowing participants to make themselves comfortable in the theatre chair and offered props to help them position their arm above the UL hardware, see Figure 2. This was to inform the ergonomic design of the final experience. Once comfortable, the participants were played six haptic sensations on to their hand. The objective was to familiarise participants with the concept of mid-air haptics and evaluate if specific haptic sensations would evoke specific emotions or ideas, especially within the context of the ocean or marine life. The mid-air sensations are represented in Figure 3. Participants were invited to comment on imagery and emotions that the sensations conveyed.

Participants were then shown two extracts from the workin-progress movie with associated haptic content: Visual Match $\left(\mathcal{V}_{\mathcal{M}}\right)$ and Audio Match $\left(\mathcal{A}_{\mathcal{M}}\right)$. $\mathcal{V}_{\mathcal{M}}$ used haptics associated with the visual imagery. For example, in a scene showing multiple fishes swimming around (Figure 5), a ripple haptic sensation (Figure 3f) could be felt to try to convey the idea of multiplicity/randomness. During $\mathcal{A}_{\mathcal{M}}$, the pulse of the haptic sensation was matched to the music soundtrack, especially moments where there was a strong bass. The clips were shown in a randomised order and the 


\begin{tabular}{cc} 
Participant & Preference \\
\hline$P_{1}$ (deaf) & neither \\
$P_{2}$ (deaf) & $\mathcal{V}_{\mathcal{M}}$ \\
$P_{3}$ (deaf) & $\mathcal{V}_{\mathcal{M}}$ \\
$P_{4}$ (deaf) & $\mathcal{V}_{\mathcal{M}}$ \\
$P_{5}$ (blind) & $\mathcal{V}_{\mathcal{M}}$ \\
$P_{6}$ (blind) & neither \\
\hline
\end{tabular}

Table 1: Reactions to video clips in WP1.

\begin{tabular}{ccc} 
Order & Ocean & Food \\
\hline$t_{0}$ & 9.5 & 6.6 \\
$t_{1}$ & 9.6 & 9.2 \\
$t_{2}$ & 9.9 & 6.9 \\
$t_{3}$ & 9.3 & 7.7 \\
Order & Energy & Water \\
\hline$t_{0}$ & 7.5 & 8.1 \\
$t_{1}$ & 9.0 & 9.0 \\
$t_{2}$ & 8.0 & 8.2 \\
$t_{3}$ & 8.1 & 8.3 \\
\hline
\end{tabular}

Table 2: Mean values from scale measurements for the four key themes.

$t_{0}$ : Before viewings

$t_{1}$ : After first viewing with haptics

$t_{2}$ : after first viewing without

haptics

$t_{3}$ : After seeing the movie twice. participants were not informed about the different haptic content approach of the clips. We wished to understand if users perceived value in either approach and if so, whether a preference existed.

Finally, participants were given an opportunity to explore the haptics with either a visual or tactile explanation of how the sensations were formed. We were interested if the participants themselves, with a better understanding of how the haptics worked, might suggest other approaches to the project.

WP2: Implementation

Eight participants, (four male and four female), ranging from 35 to 59 years old were recruited by a specialist agency on behalf of Ultraleap. Five participants were visually impaired, two of whom were blind in both eyes, the other three had sight loss that benefited from wearing glasses. Three participants were hearing impaired, all of whom benefited from hearing aid use, and did not require sign language translation. Two of the participants were wheelchair users (one manually propelled, one motorised).

This study took place at UL headquarters in Bristol (UK). A 55 inch TV screen was used to show the movie and the sound was provided through speakers embedded in the roof of the study room, see Figure 4. Participants viewed the eight-minute movie twice, (with and without haptic feedback), in a randomised order. Due to time constraints the viewings took place one after the other. A prerecorded audio description track was overlaid onto the video for the participants who were visually impaired.

We wished to establish if the implementation chosen following WP1 added value to the movie experience for the target users. To determine preference, we simply asked participants to express their favourite following the two viewings.
Beyond preference, we were also interested in whether the haptics would improve the immersive nature of the experience and, if so, whether the improved immersion would result in a greater agreement with the themes presented. Four key themes were identified in the movie: the importance of the Earth's oceans and three challenges facing humankind; producing enough clean energy, fresh water and food for a growing population. Prior to watching the movie and after each viewing, participants were asked to mark their response on an unmarked semantic differential scale to statements reflecting these themes. For example, concerning food production the statement was "Technological solutions will allow humans to grow enough food for" with the scale ranging from "A small number of people" to "The population of Earth".

To assess immersiveness participants were asked to respond to ten statements covering four measures, (temporal dissociation, focused immersion, heightened enjoyment and curiosity), associated with cognitive absorption [3]. Again, responses were marked on semantic differential scales. These, along with simple questions around movie length were used to gauge the immersiveness of the experience.

Another goal of WP2 was to gain further insight into the design of the haptic sensations. We wished to understand if particular approaches would enable us to better assign a haptic sensation to a movie scene. Participants were shown eight short clips, each of a few seconds duration, from the movie along with their corresponding haptic sensation. For example, one clip showed an aerial view of wind turbines (blades rotating, see Figure6) with the audio description "Wind turbines turn across a green landscape". The associated haptics were "rotor" illustrated Figure $3 \mathrm{~d}$. For each of the 8 clips, participants were asked to rate, on a scale from 1 to 10 , "how well did the haptic sensation match the con- 


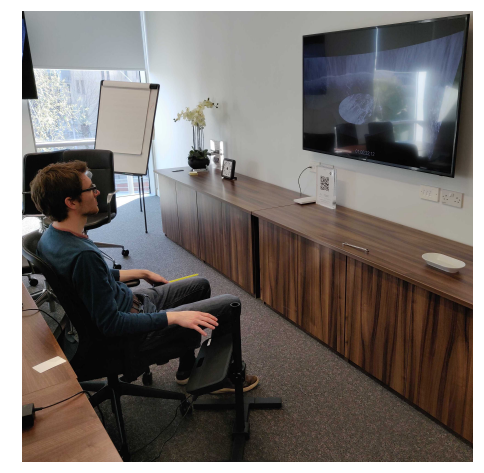

Figure 4: Setup at Ultraleap headquarters.

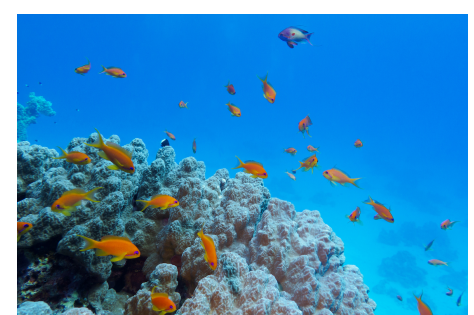

Figure 5: Illustration of a scene showing a multitude of fishes from the movie (example only).

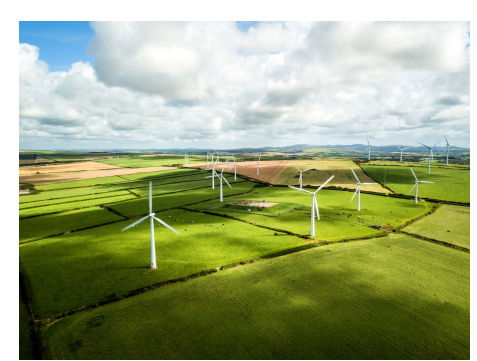

Figure 6: Illustration of a scene showing wind turbines from the movie (example only).

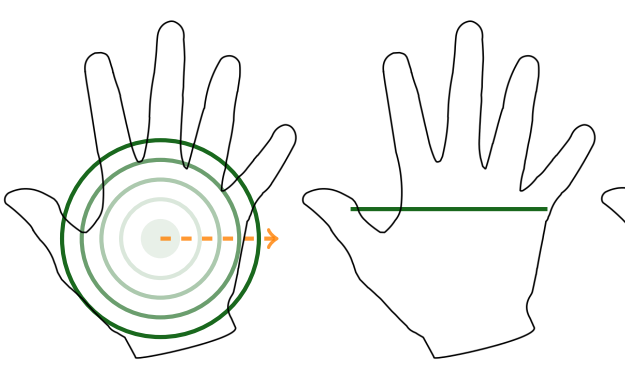

(a) Open circle

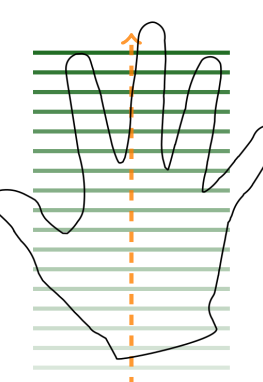

(c) Scan

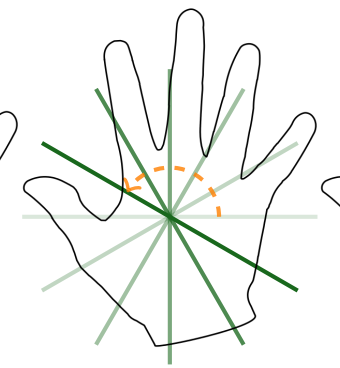

(d) Rotor

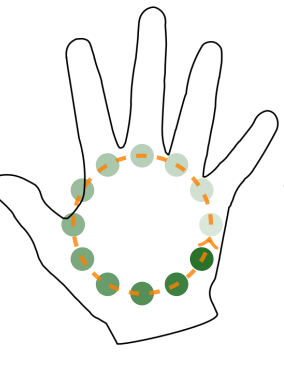

(e) Dial

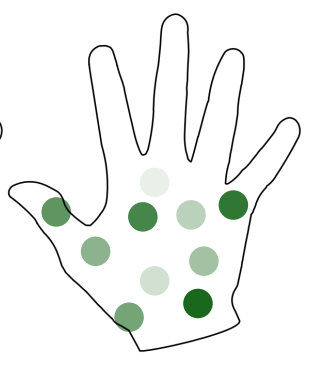

(f) Ripple

Figure 3: Illustration of the haptic sensations. The orange arrows and levels of transparency indicate movement. For example, the Open circle sensation $3 a$ initially feels like a "point" in the middle of the palm. This point then expands and users feel a sensation like a circle growing radially on their hand and fingers.

tent of the video?". The perceived links, strong and poor, were explored in open questions. The entire study was recorded to allow for further analysis.

\section{Findings}

WP1: Exploration

When the haptic sensations were played without any associated imagery, participants responded positively or neutrally to most of them. Participants were curious to explore the sensations and three people expressed associations with richer imagery.

"Open circle" and "Scan" were the two most likely to convey ocean themes. Open circle: "like a wave surge, feeling motion,like being under water"(P4) - Scan: "that feels like a wave"(P3). "Ripple" with two negative responses was the least liked: "it is a bit edgy, like I am being interrogated for something I haven't done"(P6). Interestingly the three participants who used the richest imagery in their descriptions were performers. For example, a music performer described, "Open circle" as "sort of like I am in the middle of a beautiful nature scene - it makes me think of a garden...very pleasant..very nice"(P6). As hinted in [8], individual skills or experiences may influence responses to the sensations. We were unable to conclude that a specific haptic sensation can be consistently associated with a specific theme or emotion in the absence of visual or auditory cues.

Five of the six participants perceived a value in adding haptics to video. P1 (deaf) felt the haptic sensations did not add value to either clip. P6 (blind) thought adding sensations could be helpful, but was unconvinced by either of the examples shown. He thought the $\mathcal{A}_{\mathcal{M}}$ implementation was too simplistic and not in-time with the music. Without audio description, he found it difficult to associate the sensations 


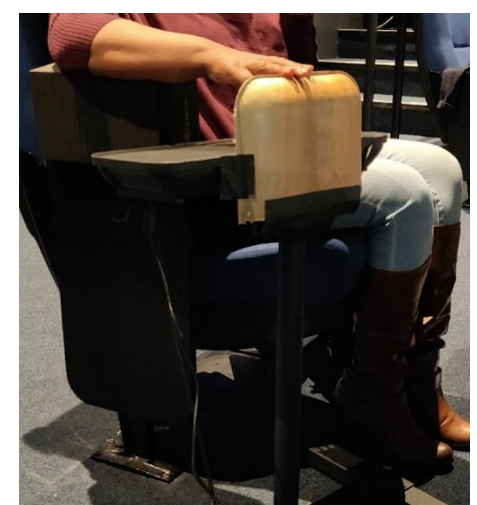

Figure 7: Improvised fingertips rest.

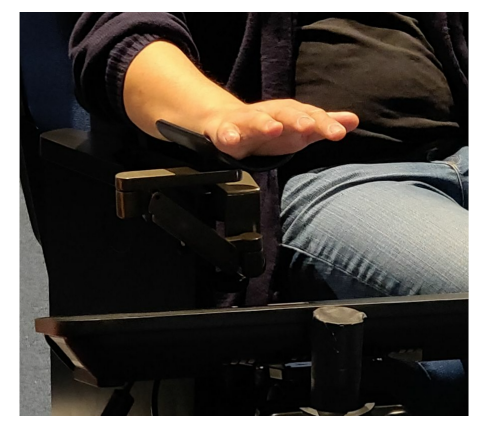

Figure 8: Improvised wrist rest.

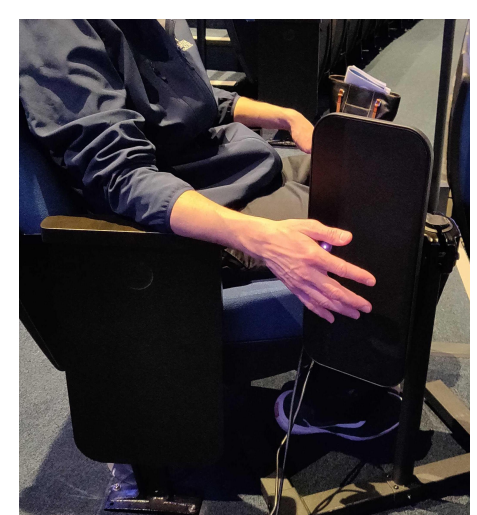

Figure 9: Idea of placing the UL array vertically. to the video during $\mathcal{V}_{\mathcal{M}}$. The four participants who enjoyed the haptics preferred $\mathcal{V}_{\mathcal{M}}$, the clip in which the sensations were associated with the visual imagery.

With such a small number of blind participants in the study, we sought additional advice from our co-author Daniel Hajas who is blind. He confirmed the requirement for an audio description. It was a useful reminder that the addition of haptics should not be seen as a replacement for other bestpractice accessibility methods. Ideally, with a longer timeline, we would have liked to have conducted more research with people who are blind. Given the time constraints, we chose the method used $\mathcal{V}_{\mathcal{M}}$ for WP2 but added audio description to link the haptics to the visuals.

The final aspect of the Exploration study concerned the ergonomics of the setup. To enjoy the haptic sensations for duration of the video ( $\approx 8 \mathrm{~min}$ ), users have to keep their hand in mid-air, around 15 to 20 centimeters above the surface of UL array. As illustrated in Figure 7 and Figure 8, we prototyped a few methods to help participants hold their hands in place. Users preferred to have their hands unconstrained and it was decided that no support other than having the option of a fingertip rest was the best solution.

The idea of using a vertical setup arose, see Figure 9. The potential benefits could be: (i) taking less space and being more comfortable alongside users' legs ; (ii) less support required for the users' hand as the wrist is less mobile in a sideways orientation; (iii) the distance between the array and the hand is more likely to be maintained if the hand drops. To assess this idea, we ran an internal user test involving six UL employees. We were surprised to discover that four out of six participants preferred the horizontal layout. This orientation was our final recommendation to the Aquarium with a reference design for a fingertip rest.
WP2: Implementation

Six out of the eight participants preferred the version of the movie with the haptic sensations. Following the two viewing one person commented, "Not as much fun...Now it was like watching a normal film...not quite as interesting or as exciting as the first time" (P8). The theme agreement results are shown in Table 2 and there is some indication that haptics influenced theme agreement. The three themes with lower agreement before watching the movie all showed higher levels of agreement following the first showing with haptics. For example, participants increased their agreement with the theme of humankind's ability to produce enough food for the planet from 6.6 to 9.2 after first seeing the movie with haptics compared to 6.9 after first seeing the movie without haptics. We also note that the average values tend to decrease at the end of the session which may be a result of boredom following two quick showings of the movie.

The cognitive absorption results are shown in Table 3 . In each case the haptic experience scored higher, most noticeably in temporal dissociation. This is consistent with responses to questions on movie length. Six of the participants said they thought the movie with haptics appeared shorter, although they could objectively tell that the movie length was the same.

The ratings of the short haptic clips, along with our discussions with the users, allowed us to draw some conclusions about sensation design. The average rankings ranged from 5.5 for the worst combination to 7.4 for the best one. Sensations with a clear relationship to on-screen movements or audio description of movements were the most successful. For example, the association between the wind turbines and the "rotor" haptic sensation worked well (7.1). One participant mentioned: "when the windmills went you could feel it". 


\begin{tabular}{lcc} 
& $\overline{\mathbf{H}}$ & $\mathbf{H}$ \\
\hline $\begin{array}{l}\text { Temporal } \\
\text { dissociation }\end{array}$ & 5.8 & 7.0 \\
\hline $\begin{array}{l}\text { Focused } \\
\text { immersion }\end{array}$ & 6.1 & 6.6 \\
\hline Enjoyment & 7.5 & 8.4 \\
\hline Curiosity & 8.5 & 9.0 \\
\hline
\end{tabular}

Table 3: Mean values for the cognitive absorption measures. H: With haptics.

$\overline{\mathbf{H}}$ Without haptics.

\section{"It was awe- some!... It felt like someone was drawing into my hand, except it was air. By the end, I could tell what was wind and what was symbolizing the fish in the sea."}

Table 4: Review from a young visitor

\section{Discussion}

This study adds to a growing body of research indicating that immersive experiences can play a role in engaging people with environmental themes. Previous work has focused on VR experiences [4] but this study begins to explore if immersive movie experiences, specifically enhanced for people with sensory impairments, can also be effective. While the nature of our study (small participant groups within which were complex differences) makes it difficult to generalise the findings more broadly, they suggest that this approach is worthy of further study. However,the authors are keen to stress the importance of also including conventional aids such as audio description and induction loop technology in any experience - mid-air haptics are an enhancement rather than replacement for such aids.

Concerning the design of haptic sensations within the context of a passive exploration, i.e. where users have their hands in a reasonably static position during the whole duration of the experience, we found that synchronising the haptic feedback and "motions" (as obvious as possible, both visually and in the audio description) seemed to be an efficient way to design compelling experiences. On the other hand, relating the haptics with on screen positions seems to be inefficient since it is hard to provide a meaningful reference to users.

The final hardware setup had the UL array and the computer running the haptic program both installed on a mobile unit that could be rolled next to any designated chair within the theatre. An optional finger rest was placed at the back of the array. We found that it was important to provide support for the hands but that users should be free to use it whenever they want during the projection of the movie. We found that a general guideline would be to keeping the experience as close to a typical movie experience as pos- sible. The integration of the haptic program to the show control solution (Medialon) used by the Aquarium proved to be straightforward in the end. The two programs were connected over the network and allowed for multiple setups to run simultaneously during a movie projection.

Beyond the study findings, we received a lot of guidance and feedback from participants. Two carers spoke to the value of reversing the normal dynamics of discussions following family entertainment experiences. They explained that often parents or siblings spend time explaining parts of an experience that might have been missed by a family member who is deaf or blind. In this case, there was a role reversal with the user of the haptic array explaining their experience to the other family members. They felt this role reversal of being the person with the "additional experience" was particularly beneficial for younger children.

\section{Conclusion \& What's next}

Overall, the project was received well, with both the inclusive design studies and final implementation reflecting well on The Aquarium. Initial feedback following installation has been positive. One young user who is blind was enthusiastic enough to send the Aquarium a review, see Figure 4.

Similar to Nanayakkara et al. [10], users were enthusiastic about the potential for haptics to communicate more musical information such as pitch, loudness, instrument types etc. Given our tight deadlines we were unable to explore this. This would be a useful area of further research.

The fact that the Aquarium could schedule this project alongside a large renovation and expansion operation implies it should be possible for other organisations to do similar work. The work was completed over a four month period and to schedule. We hope this paper encourages others to 
do similar projects, exploring innovative solutions to make entertainment and educational experiences more inclusive.

\section{Acknowledgements}

This project would not have been possible without the vision and determination of the Aquarium of the Pacific. In particular we would like to thank Fahria Qader and Derek Balsillie who patiently supported and accommodated this work, while managing a large and complex building project.

\section{REFERENCES}

[1] . www.aquariumofpacific.org. ( ).

[2] Damien Ablart, Carlos Velasco, and Marianna Obrist. 2017. Integrating Mid-Air Haptics into Movie

Experiences. In Proceedings of the 2017 ACM International Conference on Interactive Experiences for TV and Online Video (TVX '17). ACM, New York, NY, USA, 77-84. DOI :

http://dx.doi.org/10.1145/3077548.3077551

[3] Ritu Agarwal and Elena Karahanna. 2000. Time flies when you're having fun: Cognitive absorption and beliefs about information technology usage. MIS quarterly (2000), 665-694.

[4] Sun Joo Ahn, Joshua Bostick, Elise Ogle, Kristine L Nowak, Kara T McGillicuddy, and Jeremy N Bailenson. 2016. Experiencing nature: Embodying animals in immersive virtual environments increases inclusion of nature in self and involvement with nature. Journal of Computer-Mediated Communication 21, 6 (2016), 399-419.

[5] William Frier, Damien Ablart, Jamie Chilles, Benjamin Long, Marcello Giordano, Marianna Obrist, and Sriram
Subramanian. 2018. Using spatiotemporal modulation to draw tactile patterns in mid-air. In International Conference on Human Haptic Sensing and Touch Enabled Computer Applications. Springer, 270-281.

[6] Matthew J Hertenstein, Rachel Holmes, Margaret McCullough, and Dacher Keltner. 2009. The communication of emotion via touch. Emotion 9, 4 (2009), 566

[7] M Gail Jones, James Minogue, Tom Oppewal, Michelle P Cook, and Bethany Broadwell. 2006. Visualizing without vision at the microscale: Students with visual impairments explore cells with touch. Journal of science education and technology 15, 5-6 (2006), 345-351.

[8] Richard E Mayer. 2003. The promise of multimedia learning: using the same instructional design methods across different media. Learning and instruction 13, 2 (2003), 125-139.

[9] Chang S Nam, Yueqing Li, Takehiko Yamaguchi, and Tonya L Smith-Jackson. 2012. Haptic user interfaces for the visually impaired: Implications for haptically enhanced science learning systems. International Journal of Human-Computer Interaction 28, 12 (2012), 784-798.

[10] Suranga Nanayakkara, Elizabeth Taylor, Lonce Wyse, and S H Ong. 2009. An enhanced musical experience for the deaf: design and evaluation of a music display and a haptic chair. In Proceedings of the SIGCHI Conference on Human Factors in Computing Systems. ACM, 337-346. 